\title{
Factores asociados a reingreso hospitalario precoz posterior a resecciones hepáticas y pancreáticas
}

\author{
Rodrigo Tejos S. ${ }^{1}$, Alejandro Rojas C. ${ }^{1}$, Rolando Rebolledo A. ${ }^{2}$, \\ Pablo Achurra T. ${ }^{2}$, Sergio Pacheco S. ${ }^{2}$, Eduardo Briceño V. ${ }^{2}$, \\ Nicolás Jarufe C. ${ }^{2}$, Jorge Martínez C. ${ }^{2}$ y Juan Francisco Guerra C. ${ }^{2}$
}

\section{Hospital readmission rates after hepatic and pancreatic resections}

\begin{abstract}
Aim: To determine readmission rates and its associated factors in patients undergoing pancreatic and hepatic resections at our center. Matherial and Methods: Perioperative variables of patients undergoing pancreatic and hepatic resections between 2012-2014 were reviewed. Demographic and perioepartive data, as well as up -to postoperative day 30 - readmisson rates were analyzed. Emergency cases, postoperative mortality and/or patients undergoing less extensive surgery (less than 2 Couinaud's segments, non-anatomical resections and non resective cases such as bilioenteric anastomoses) were excluded. Readmission associated factors were identified using both univariate $\left(\chi^{2}\right.$ for categorical and t-student's/Mann-Whitney for continuous variables) and multivariate (logistic regression) analysis. Results: 116 cases were included, $50.9 \%$ female. Mean postoperative stay was 14 days. Pancreatic adenocarcinoma was the most frequent diagnosis (25.9\%), and the $40.5 \%$ of surgical procedures were pancreaticoduodenectomy. Overall 30 -day readmission rate was $18.1 \%$, with a $23.7 \%$ for pancreatic resections and $12.2 \%$ for hepatic resections. According to univariate analysis; readmission associated factors were: pancreatic resection, preoperative White cell count, the development of postoperative complications and postoperative length of stay. On Multivariate analysis only postoperative stay was the only significant associate factor [OR 1,2 CI 95\% 1.1- $1.5(p=0.001)]$. Conclussion: Readmission rates after pancreatic and hepatic resections are elevated, even in high-volume centers. Postoperative length of stay is a consistent risk factor for readmission after these type of surgeries. We highly recommend including this parameter as a quality marker of our surgical results in our country.
\end{abstract}

Key words: patient readmission; pancreas; pancreatectomy; pancreaticoduodenectomy; hepatectomy.

\section{Resumen}

Objetivo: Determinar la frecuencia de reingreso hospitalario y sus factores asociados en pacientes sometidos a resecciones hepáticas o pancreáticas en nuestro centro. Metodología: Se revisaron registros de pacientes sometidos a resecciones hepáticas o pancreáticas entre 2012 y 2014. Se registraron variables biodemográficas, quirúrgicas y reingresos hasta 30 días posalta. Se excluyó la cirugía de urgencia, pacientes fallecidos durante la hospitalización, pacientes sometidos a resecciones hepáticas menores a dos segmentos, cirugías no anatómicas o cirugía no resectiva. El análisis estadístico univariable se realizó con prueba $\chi^{2}$ para variables categóricas y T Student/Mann Whitney para variables continuas. El análisis multivariable se realizó con regresión logística. Resultados: Se incluyeron 116 pacientes, $50,9 \%$ mujeres. La estadía promedio fue de 14 días. El adenocarcinoma pancreático fue el diagnóstico más frecuente $(25,9 \%)$ y $40,5 \%$ de los procedimientos quirúrgicos fueron pancreatoduodenectomías. La tasa de reingreso global fue $18,1 \%$, mayor en pancreatectomías respecto a hepatectomías $(23,7 \mathrm{vs}$ $12,2 \%$ respectivamente $\mathrm{p}<0,05$ ). Los factores asociados a reingreso fueron: resección de páncreas, leucocitos preoperatorios, complicaciones posoperatorias y tiempo de estadía hospitalaria. Tras el análisis multivariable, sólo el tiempo hospitalario se asocia de forma independiente al reingreso precoz [OR
${ }^{1}$ Facultad de Medicina, Pontificia Universidad Católica de Chile. Santiago, Chile. ${ }^{2}$ Departamento de Cirugía Digestiva. Facultad de Medicina, Pontificia Universidad Católica de Chile, Santiago, Chile.

Recibido el 29 de octubre de 2017 y aceptado para publicación el 19 de diciembre de 2017.

Correspondencia a: Dr. Juan Francisco Guerra jfguerra@med.puc.cl 
1,2 IC 95\% 1,1-1,5 $(\mathrm{p}=0,001)]$. Conclusión: La estadía hospitalaria prolongada es un factor de riesgo consistente en la literatura para la rehospitalización posterior a resecciones hepáticas o pancreáticas. La tasa de reingreso posterior a resecciones hepáticas o pancreáticas es elevada, incluso en centros de alto volumen. Recomendamos el uso de este parámetro como un nuevo instrumento de medición de calidad en los resultados quirúrgicos en nuestro país.

Palabras clave: reingreso hospitalario; cirugía hepática; cirugía pancreática; hepatectomía; pancreatoduodenectomía.

\section{Introducción}

El reingreso hospitalario precoz (RHP) es aquel que ocurre dentro de los primeros 30 días posteriores al alta ${ }^{1}$. Este evento se ha convertido en un problema para el funcionamiento de los servicios de salud, ya que genera costos elevados asociados a prestaciones médicas potencialmente evitables ${ }^{2}$. Por esta razón, se ha establecido como un marcador de calidad e incluso algunos países han instaurado sanciones económicas a prestadores con indicadores deficientes ${ }^{3}$. Sumado a las desventajas económicas que produce, el RHP se asocia a un aumento de la morbilidad y mortalidad en los pacientes que la presentan, sobre todo en cirugías de alta complejidad como es el caso de la cirugía hepática y pancreática ${ }^{4}$. Diversos autores han estudiado la incidencia y factores asociados a este evento, con prevalencias globales de RHP descritas entre $14,2 \%$ y $20,9 \%^{1,4-8}$. Los principales factores de riesgo asociados al RHP son el número y la severidad de las comorbilidades del paciente, la presencia de complicaciones posoperatorias y el tiempo de estadía hospitalaria ${ }^{4-8}$.

El objetivo principal de este estudio fue determinar la frecuencia de RHP en una cohorte de pacientes sometidos a cirugía de resección hepática o pancreática en nuestro Centro.

\section{Material y Métodos}

Estudio de cohorte histórica. Se revisaron las fichas clínicas e informáticas de pacientes operados entre junio de 2012 y junio de 2014 por el equipo de cirugía hepatobiliar y pancreática del Hospital Clínico de la Pontificia Universidad Católica (PUC). Se incluyeron en el estudio todos los pacientes sometidos a cirugías de resección hepática y pancreática mayor; definidas como mayores a dos segmentos de Couinaud en el caso de las hepatectomías y a resecciones pancreáticas formales (duodenopancreatectomías y pancreatectomías corporocaudales). Se excluyeron las cirugías de urgencia, resecciones "no anatómicas" y pacientes con información incompleta o que fallecieron durante la hospitalización. Se registraron variables de laboratorio, clínicas y quirúrgicas. Las complicaciones posoperatorias se categorizaron según la clasificación Clavien-Dindo: grados 1 y 2 se definieron como complicaciones menores, mientras que grado 3 y 4 se consideraron complicaciones mayores ${ }^{7}$.

El evento o outcome principal analizado fue el reingreso de los pacientes dentro de los primeros 30 días posteriores a su alta. En este grupo se analizaron, además, las características propias que motivaron el reingreso, así como morbilidad, mortalidad y re-intervenciones quirúrgicas. Los pacientes operados que no presentaron RHP fueron considerados como "grupo control".

El análisis estadístico se realizó mediante el programa SPSS versión 17 (2009, Chicago IL, USA). Para la descripción de variables continuas se utilizaron los estadígrafos media y desviación estándar o mediana e intervalo, según correspondiera. La descripción de variables categóricas se realizó mediante frecuencias absolutas y porcentajes.

Se utilizó prueba $\chi^{2} \mathrm{y} t$ de student/Mann-whitney para variables categóricas o continuas, respectivamente. Se utilizó regresión logística para el análisis multivariable. Se consideró significativo un valor de $\mathrm{p}<0,05$.

Este estudio fue aprobado por el Comité de Ética de Investigación de la Escuela de Medicina de la Pontificia Universidad Católica de Chile.

\section{Resultados}

Un total de 140 pacientes fueron sometidos a resecciones hepáticas o pancreáticas entre los años 2012 y 2014. Luego de aplicar los criterios de exclusión, se revisaron las fichas clínicas de 116 pacientes. La Tabla 1 resume las características de la población general y de los grupos generados al categorizar a los pacientes según RHP. El grupo que 
Tabla 1. Características de la población

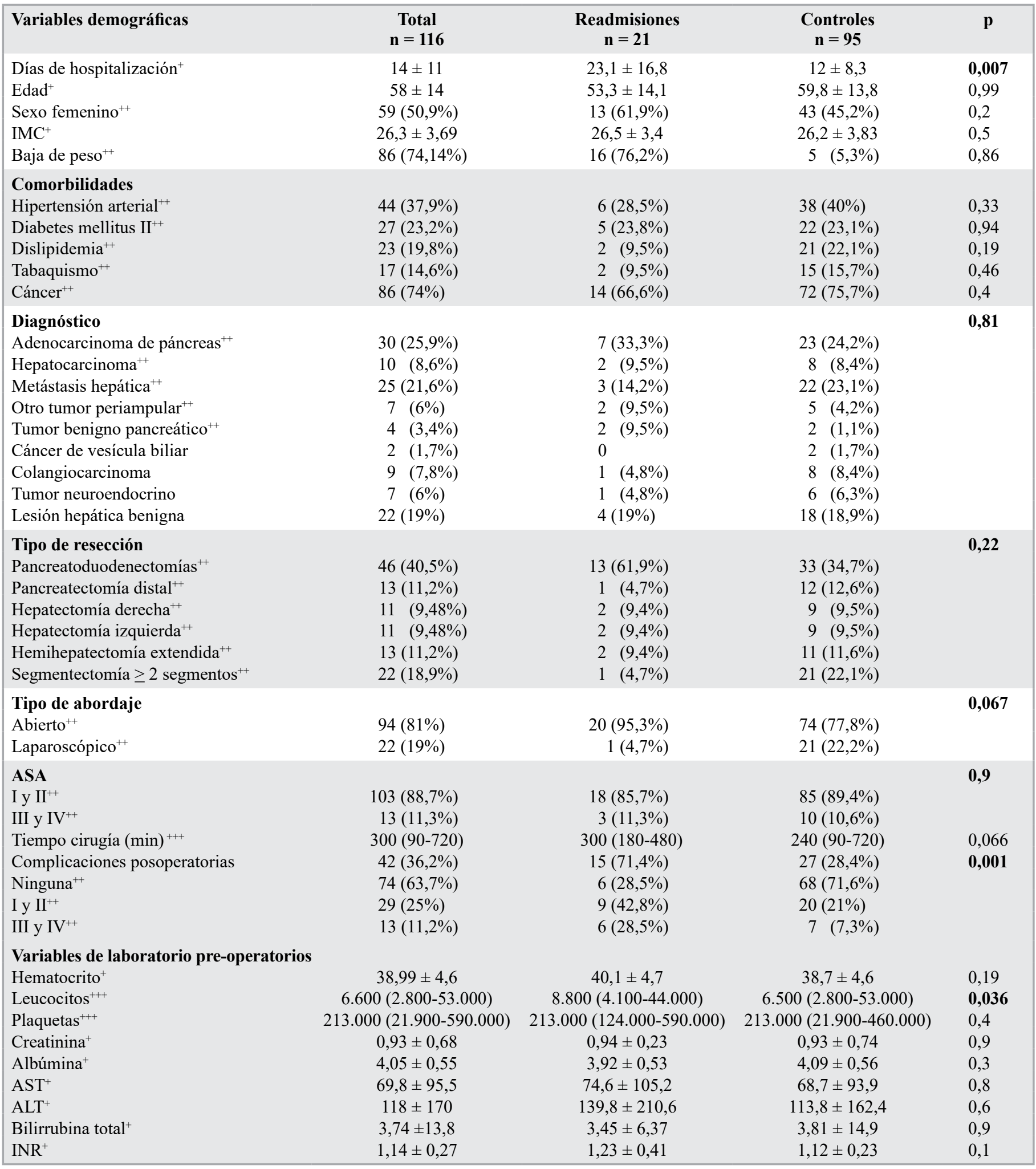

${ }^{+}$Promedio \pm Desviación estándar. ${ }^{++}$n/porcentaje. ${ }^{+++}$Mediana/Intervalo. 


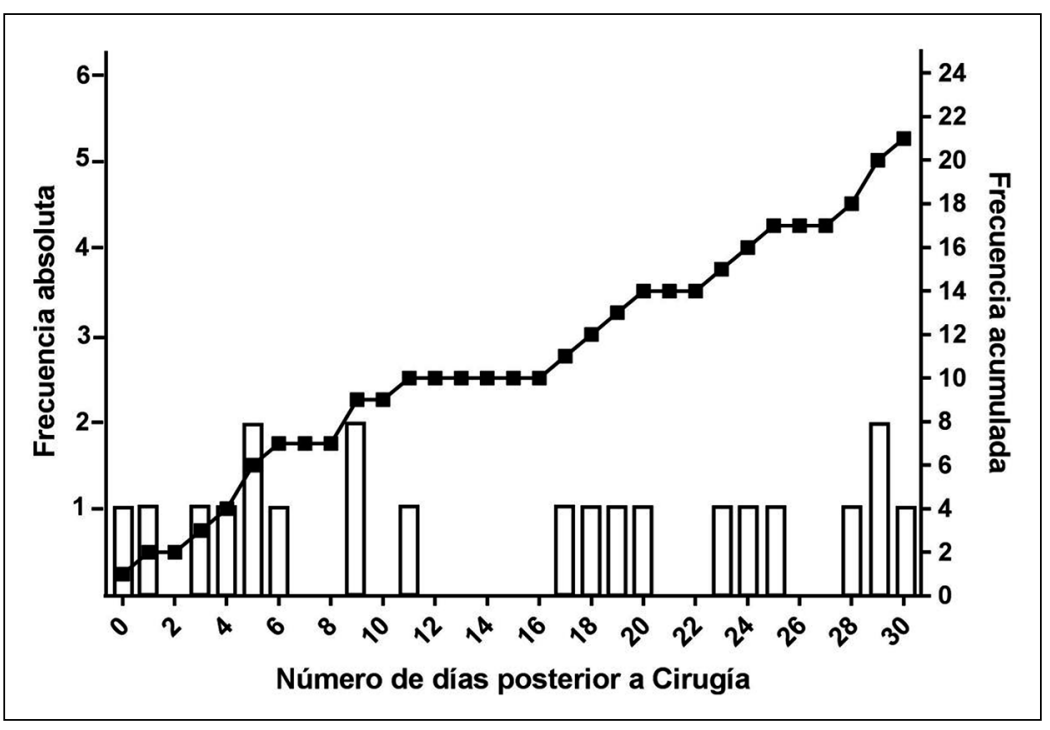

Figura 1. Distribución de distribución temporal del reingreso hospitalario precoz.

Tabla 2. Características de readmisión a 30 días

\section{Características}

Readmisión hospitalaria global

Readmisión en cirugía pancreática $(\mathrm{n}=59)$

Readmisión en cirugía hepática $(\mathrm{n}=57)$

$21(18,1 \%)^{+}$

$14(23,7 \%)^{+}$

$7(12,2 \%)^{-}$

Motivo de consulta en Servicio de Urgencias

Dolor abdominal

$12(57 \%)^{+}$

Fiebre

$3(14 \%)^{+}$

Otros

$6(29 \%)^{+}$

Diagnóstico de reingreso

Colección intraabdominal

$9(43 \%)^{+}$

Infección de herida operatoria

$2(9,5 \%)^{+}$

Pancreatitis

$1(4,7 \%)$

Colangitis

$1(4,7 \%)^{+}$

Otros

$8(38 \%)^{+}$

Estadía hospitalaria en reingreso (días)

$6(3-30)^{+}$

Reintervenciones

$4(19 \%)$

$2(50 \%)$

Punción percutánea

$1(25 \%)$

Drenaje absceso prostático transrrectal

$1(25 \%)$

Mortalidad

0

${ }^{+}$n/porcentaje. ${ }^{++}$Mediana/intervalo.

Tabla 3. Análisis multivariable

\begin{tabular}{|lccc|}
\hline Variable & OR & IC 95\% OR & Valor $\mathbf{~ p}$ \\
\hline Estadía hospitalaria & 1,2 & $1,1-1,5$ & $\mathbf{0 , 0 0 1}$ \\
Sexo & & & 0,095 \\
Leucocitos preoperatorios & & & 0,099 \\
Complicaciones posoperatorias & & & 0,06 \\
\hline Tipo de resección & & & 0,6 \\
\hline
\end{tabular}

no presentó RHP fue considerado como control. El promedio de edad de la población general fue de 58 +14 años, con leve predominio de sexo femenino $(50,9 \%)$ y la estadía hospitalaria promedio fue de $14+11$ días. El IMC promedio de la población fue de $26,3+3,69 \mathrm{~kg}$, mientras que la hipertensión fue la comorbilidad más frecuente. Ochenta y seis pacientes (74\%) tenía el diagnóstico de cáncer, siendo el adenocarcinoma pancreático el diagnóstico más frecuente $(25,9 \%)$, seguido por metástasis hepáticas 25 (21,6\%). La mayoría de los pacientes fueron clasificados como ASA I o II $103(88,7 \%)$ y la cirugía más frecuente fue la pancreatoduodenectomía 46 casos $(40,5 \%)$. El abordaje abierto fue el más empleado en esta serie 94 (81\%) y el tiempo promedio de intervención fue $304,5+89,2 \mathrm{~min}$. Se presentaron complicaciones en 42 (36,3\%) de los pacientes y en caso de presentarlas, la mayoría fueron menores 29 (69\%).

\section{Análisis de reingreso}

Un total de 21 pacientes presentaron RHP $(18,1 \%)$, con una mediana de 17 días para la aparición del evento. La distribución temporal del RHP se presenta en La Figura 1. La frecuencia de reingreso fue mayor en resecciones de páncreas, comparado con hepatectomías $(23,7 \%$ vs $12,2 \%$ respectivamente $\mathrm{p}<0,05)$. Los síntomas que más frecuentemente motivaron el RHP fueron el dolor abdominal $57 \%$ (12) y fiebre $14 \%$ (3). Los diagnósticos más frecuentes de reingreso fueron colección intraabdominal (9 casos) e infección de la herida operatoria (2 pacientes). La mediana de estadía hospitalaria debido al RHP fue 6 (3-30) días. En esta serie no se registró mortalidad. Cuatro pacientes presentaron complicaciones que requirieron un manejo específico; en 2 casos se realizó una punción percutánea radiológica, un paciente requirió drenaje transrrectal (absceso prostático) y un paciente fue sometido a un aseo quirúrgico por vía laparoscópica. Las características de este grupo se resumen en la Tabla 2.

\section{Factores asociados al reingreso}

De acuerdo al análisis univariable (Tabla 1), se observa que el tiempo de estadía en la intervención original $(\mathrm{p}=0,01)$, leucocitos preoperatorios $(\mathrm{p}=0,036)$ y la presencia de complicaciones posoperatorias $(\mathrm{p}=0,001)$, presentan diferencias significativas entre los pacientes que presentaron RHP y el grupo control. Al realizar el análisis multivariable (Tabla 3) se observa que sólo la duración de la estadía hospitalaria se asocia de forma independiente con la posibilidad de reingreso a 30 días, con un OR de 1,2 [IC 95\% 1,1-1,5 (p<0,001)]. 


\section{Discusión}

El reingreso hospitalario no planificado de pacientes es un evento frecuente en los centros hospitalarios de todo el mundo y afecta de forma transversal a todas las especialidades ${ }^{2,3,9-11}$. Su principal importancia radica en el aumento de costos económicos y en peores resultados de salud para los pacientes que la presentan ${ }^{2}$. Existen distintas definiciones en el tiempo transcurrido desde el egreso hospitalario hasta que el paciente se rehospitaliza, siendo más frecuentemente utilizadas la aparición de este evento a 30 y 90 días ${ }^{7,8,12-14}$. Se utilizó la definición de RHP como la rehospitalización de pacientes dentro de los primeros 30 días posteriores a su alta, con el fin de comparar nuestros resultados con series internacionales, las cuales han demostrado que el mayor porcentaje de eventos se produce en este período $(>90 \%)^{15-19}$.

La cifra de RHP global en resecciones hepáticas y pancreáticas ha sido consistentemente elevada en comparación a otros tipos de cirugía, como la cirugía bariátrica o colorrectal, donde se reportan cifras entre $3,1-7,2 \%$ y $7-15 \%$ respectivamente ${ }^{20-26}$. Si bien la complejidad técnica y menor reserva funcional de los pacientes sometidos a este tipo de resecciones explican gran parte de esta cifra, es necesario entender las causas del RHP para crear estrategias preventivas. En este sentido, existen claras diferencias en las frecuencias de rehospitalización según el tipo de resección quirúrgica realizada. Tanto en el presente estudio, como en publicaciones previas, la frecuencia de este evento fue mayor en las resecciones pancreáticas que en las hepáticas ${ }^{4,6}$. Si bien el RHP de resecciones hepáticas encontradas en este estudio fue similar a lo publicado previamente (12,2 vs 12,7-15,5\% $)^{4,5,8,27}$, el RHP de resecciones pancreáticas fue levemente superior a centros internacionales $(23,7 \% \text { vs } 16-21,3 \%)^{7,28,29}$. Creemos que el elevado número de cirugías anuales que realizan estos Centros probablemente explica la diferencia, ya que se ha demostrado la influencia positiva del volumen quirúrgico en los resultados posoperato$\operatorname{rios}^{7,30,31}$.

Dentro de los principales factores de riesgo descritos para el RHP para resecciones hepáticas y pancreáticas se encuentran el número y la severidad de las comorbilidades del paciente, la presencia de complicaciones posoperatorias y el tiempo de estadía hospitalaria ${ }^{4,6}$.

Es interesante la presencia de leucocitos preoperatorios encontrados durante nuestra investigación como un factor asociado al RHP en el análisis univariable, ya que esto no había sido reportado previamente. No existe un análisis de parámetros de laboratorio preoperatorios en los trabajos previamente publicados, por lo que creemos que se deben realizar más estudios para analizar dicha asociación.

En nuestro análisis multivariable sólo el tiempo de estadía hospitalaria se mantuvo como un factor de riesgo independiente asociado a la probabilidad de presentar RHP. Otros factores de riesgo descritos como las complicaciones posoperatorias si bien mostraron asociación en el análisis univariable, no lograron mantener su efecto al ajustar por variables confundentes. Pese a esto, creemos que es importante destacar que un $81,5 \%$ de los pacientes que reingresaron presentó algún tipo de complicación, aunque la mayoría de estas fue menor (Clavien I-II).

Pese a la relevancia internacional del RHP como marcador de calidad en cirugía, hasta la fecha ha sido poco utilizado en nuestro país y sólo un estudio en cirugía colorrectal ha reportado este evento ${ }^{26}$. Dado el impacto del RHP en la morbimortalidad de los pacientes, consideramos necesario el reporte, seguimiento y análisis de este evento en los distintos centros de nuestro país. Mejorar el conocimiento del RHP en el escenario nacional podría permitir generar estrategias preventivas orientadas a nuestra población. Además, creemos que este evento podría convertirse en un marcador de calidad de los distintos centros nacionales, al reflejar de forma concreta los resultados quirúrgicos a corto plazo.

Las principales limitaciones de nuestro trabajo radican en su diseño retrospectivo, ya que los resultados obtenidos dependen directamente de la calidad de los registros clínicos de las fichas analizadas. Pese a esto, el presente trabajo es el primer reporte nacional en analizar la re-hospitalización de pacientes sometidos a resecciones hepáticas o pancreáticas y creemos que nuestros resultados son susceptibles de ser incorporados en un metaánalisis que incluya un mayor número de pacientes, con el fin de obtener conclusiones que puedan ser generalizadas a la práctica clínica habitual.

\section{Conclusión}

La tasa de reingreso posterior a resecciones hepáticas o pancreáticas es elevada, incluso en centros de alto volumen. La estadía hospitalaria prolongada durante la intervención inicial es un factor de riesgo consistente en la literatura para la rehospitalización posterior a resecciones hepáticas o pancreáticas. Recomendamos el uso de este parámetro como un nuevo instrumento para la medición de la calidad de los resultados quirúrgicos en nuestro país. 


\section{Responsabilidades éticas}

Protección de personas y animales. Los autores declaran que para esta investigación no se han realizado experimentos en seres humanos ni en animales.

Confidencialidad de los datos. Los autores declaran que han seguido los protocolos de su centro de trabajo sobre la publicación de datos de pacientes.

Derecho a la privacidad y consentimiento informado. Los autores declaran que en este artículo no aparecen datos de pacientes.

\section{Conflicto de intereses}

Los autores declaran que no tienen conflictos de interés.

\section{Financiación}

Este estudio fue financiado con fondos del Departamento de Cirugía Digestiva de la facultad de Medicina de la Pontificia Universidad Católica de Chile.

\section{Bibliografía}

1. Sutton J, Wilson G, Wima K, Hoehn R, Cutler-Quillin R, Hanseman

$\mathrm{D}$, et al. Readmission After

Pancreaticoduodenectomy: The Influence of the Volume Effect Beyond Mortality. Annals of surgical oncology 2015;22:3785-92.

2. Jencks S, Williams M, Coleman E. Rehospitalizations among patients in the Medicare fee-for-service program. The New England journal of medicine 2009;360:1418-28.

3. Hansen L, Young R, Hinami K, Leung A, Williams M. Interventions to reduce 30-day rehospitalization: a systematic review. Annals of internal medicine 2011;155:520-8.

4. Schneider E, Hyder O, Wolfgang C, Hirose K, Choti M, Makary M, et al. Patient readmission and mortality after surgery for hepato-pancreato-biliary malignancies. Journal of the American College of Surgeons 2012;215:607-15.

5. Spolverato G, Ejaz A, Kim Y, Weiss M, Wolfgang CL, Hirose K, et al. Readmission incidence and associated factors after a hepatic resection at a major hepato-pancreatico-biliary academic centre. HPB 2014;16: 972-8.

6. Lucas D, Sweeney J, Pawlik T. The timing of complications impacts risk of readmission after hepatopancreatobiliary surgery. Surgery 2014;155:945-53.

7. Hyder O, Dodson R, Nathan H, Schneider E, Weiss M, Cameron J, et al. Influence of patient, physician, and hospital factors on 30-day readmission following pancreatoduodenectomy in the United
States. JAMA surgery 2013;148:1095102.

8. Kelly K, Iannuzzi J, Rickles A, Monson J, Fleming FJ. Risk factors associated with 30-day postoperative readmissions in major gastrointestinal resections. Journal of gastrointestinal surgery 2014; 18:35-43.

9. Kronzer V, Ben Abdallah A, McKinnon S, Wildes T, Avidan M. Ability of preoperative falls to predict postsurgical outcomes in non-selected patients undergoing elective surgery at an academic medical centre: protocol for a prospective cohort study. BMJ open 2016;6: 011570.

10. Noyes K, Baack-Kukreja J, Messing E, Schoeniger L, Galka E, Pan W, et al. Surgical readmissions: results of integrating pre-, peri- and postsurgical care. Nursing open 2016;3:168-78.

11. Robinson R. The HOSPITAL score as a predictor of 30 day readmission in a retrospective study at a university affiliated community hospital. PeerJ. 2016;4:e2441. doi: 10.7717/peerj.2441. eCollection 2016.

12. Cerullo M, Gani F, Chen S, Canner J, Pawlik T. Readmission after major surgery: effect of the postdischarge environment. The Journal of surgical research 2016;205:318-26.

13. Mise Y, Vauthey J, Zimmitti G, Parker $\mathrm{N}$, Conrad C, Aloia T, et al. Ninety-day Postoperative Mortality Is a Legitimate Measure of Hepatopancreatobiliary Surgical Quality. Annals of surgery 2015;262:1071-8.

14. Stitzenberg K, Chang Y, Smith A, Meyers $M$, Nielsen M. Impact of Location of
Readmission on Outcomes After Major Cancer Surgery. Annals of surgical oncology. 2016. DOI: 10.1245/s10434016-5528-1 [Epub ahead of print]

15. Bagante F, Spolverato G, Strasberg S, Gani F, Thompson V, Hall B, et al. Minimally Invasive $v s$. Open Hepatectomy: a Comparative Analysis of the National Surgical Quality Improvement Program Database. Journal of gastrointestinal surgery 2016;20:160817.

16. Ceppa E, Pitt H, Nakeeb A, Schmidt C, Zyromski N, House M, et al. Reducing Readmissions after Pancreatectomy: Limiting Complications and Coordinating the Care Continum. Journal of the American College of Surgeons 2015;221:708-16.

17. Kariv Y, Wang W, Senagore A, Hammel J, Fazio V, Delaney C. Multivariable analysis of factors associated with hospital readmission after intestinal surgery. American journal of surgery 2006;191:364-71.

18. Mosquera C, Vohra N, Fitzgerald T, Zervos E. Discharge with Pancreatic Fistula after Pancreaticoduodenectomy Independently Predicts Hospital Readmission. The American surgeon 2016;82:698-703.

19. Sadot E, Brennan M, Lee S, Allen P, Gönen M, Groeger J, et al. Readmission after pancreatic resection: causes and causality pattern. Annals of surgical oncology 2014;21:4342-50.

20. Francis N, Mason J, Salib E, Allanby L, Messenger D, Allison A, et al. Factors predicting 30-day readmission after laparoscopic colorectal cancer surgery 
ARTÍCULO ORIGINAL

within an enhanced recovery programme. Colorectal disease 2015; 17:0148-54.

21. Garg T, Rosas U, Rogan D, Hines H, Rivas H, Morton J, et al. Characterizing Readmissions After Bariatric Surgery. Journal of gastrointestinal surgery: 2016 Sep 9. DOI: 10.1007/s11605-016-3247-3 [Epub ahead of print].

22. Hendren S, Morris A, Zhang W, Dimick J. Early discharge and hospital readmission after colectomy for cancer. Diseases of the colon and rectum 2011;54:1362-7.

23. Kiran R, Delaney C, Senagore A, Steel M, Garafalo T, Fazio V. Outcomes and prediction of hospital readmission after intestinal surgery. Journal of the American College of Surgeons 2004; 198:877-83

24. Saunders J, Ballantyne G, Belsley S, Stephens D, Trivedi A, Ewing D, et al. 30-day readmission rates at a high volume bariatric surgery center: laparoscopic adjustable gastric banding, laparoscopic gastric bypass, and vertical banded gastroplasty-Roux-en-Y gastric bypass. Obesity surgery 2007;17:1171-7.

25. Schneider E, Hyder O, Brooke B, Efron J, Cameron J, Edil B, et al. Patient readmission and mortality after colorectal surgery for colon cancer: impact of length of stay relative to other clinical factors. Journal of the American College of Surgeons 2012;214:390-8.

26. Bannura G, Gallardo C, Vargas C, Barrera A, Melo C, Illanes F. Readmission rates in complex colorrectal surgery. Rev Chil Cir. 2015;67:393-8.

27. Barbas A, Turley R, Mallipeddi M, Lidsky M, Reddy S, White R, et al. Examining reoperation and readmission after hepatic surgery. Journal of the American College of Surgeons 2013;216:915-23.

28. Reddy D, Townsend C, Kuo Y, Freeman $\mathrm{J}$, Goodwin J, Riall T. Readmission after pancreatectomy for pancreatic cancer in Medicare patients. Journal of gastrointestinal surgery: 2009;13:1963-74.

29. Ahmad S, Edwards M, Sutton J, Grewal S, Hanseman D, Maithel S, et al. Factors influencing readmission after pancreaticoduodenectomy: a multiinstitutional study of 1302 patients. Annals of surgery 2012;256:529-37.

30. Hata T, Motoi F, Ishida M, Naitoh T, Katayose Y, Egawa S, et al. Effect of Hospital Volume on Surgical Outcomes After Pancreaticoduodenectomy: A Systematic Review and Meta-analysis. Annals of surgery 2016;263:664-72. 